\title{
Caracterização do desempenho funcional de indivíduos com síndrome de Down
}

\section{Characterization of functional performance in people with Down syndrome}

\author{
Aline Bernardes de Souza ${ }^{1}$, Silvana Maria Blascovi-Assis ${ }^{2}$, Luciana Krauss Rezende ${ }^{3}$, \\ Raquel Cymrot ${ }^{4}$
}

http://dx.doi.org/10.11606/issn.2238-6149.v26i1p102-108

Souza AB, Blascovi-Assis SM, Rezende LK, Cymrot R. Caracterização do desempenho funcional de indivíduos com síndrome de Down. Rev Ter Ocup Univ São Paulo. 2015 jan./ abr.;26(1):102-8.

RESUMO: Esta pesquisa procurou caracterizar o desempenho de crianças e jovens com síndrome de Down e a interação entre o perfil funcional e a assistência prestada pelo cuidador. Participaram desta pesquisa 44 cuidadores de crianças e jovens com síndrome de Down, com idades entre 7 anos e 6 meses de idade a 15 anos incompletos que foram avaliados através do Inventário PEDI. Estas crianças e jovens com SD apresentaram um desempenho inferior em relação ao padrão normal além de menores escores na função social. Observou-se a influência da idade sobre algumas áreas funcionais e a linearidade entre a evolução da capacidade funcional e da ajuda do cuidador. Notou-se, também, que as crianças e jovens com síndrome de Down apresentam baixo desempenho funcional nesta faixa etária sob influência da idade.

DESCRITORES: Síndrome de Down; Atividades cotidianas; Avaliação.
Souza AB, Blascovi-Assis SM, Rezende LK, Cymrot R. Characterization of functional performance in people with Down syndrome. Rev Ter Ocup Univ São Paulo. 2015 jan./ abr.;26(1):102-8.

ABSTRACT: This survey sought to characterize the performance of children and young people with DS and the interaction between their functional profile and the assistance provided by the caregiver. Participated in the research 44 caregivers of children and young people with DS, aged between 7 years and 6 months old to 15 years old incomplete. They were assessed through PEDI. These children and young people with DS presented lower functional performance compared to normal pattern and had lower scores in social function. It was observed the influence of age on some functional areas and the linearity between the evolution of functional capacity and the assistance of caregiver. It was also noted that children and young people with DS present low functional performance in this age group under age influence.

KEYWORDS: Down syndrome; Everyday activities; Evaluation.

\footnotetext{
Este trabalho é parte integrante da dissertação de mestrado da primeira autora.

${ }^{1}$ Doutoranda em Distúrbios do Desenvolvimento, Universidade Presbiteriana Mackenzie, São Paulo, SP, Brasil.

${ }^{2}$ Docente do Programa de Pós-Graduação em Distúrbios do Desenvolvimento, Universidade Presbiteriana Mackenzie, São Paulo, SP, Brasil.

${ }^{3}$ Pós-doutoranda em Desenvolvimento Tecnologias e Sociedade, Universidade Federal de Itajubá, Itajubá, MG, Brasil.

${ }^{4}$ Docente do Curso de Engenharia, Universidade Presbiteriana Mackenzie, São Paulo, SP, Brasil.

Endereço para correspondência: Aline Bernardes de Souza. Rua Julio Baumgarten, n. 512 - apto 212. Bairro Vila Nova. CEP: $89037-010$. Blumenau, SC, Brasil. E-mail: alinebernardessouza@yahoo.com.br.
} 


\section{INTRODUÇÃO}

$\Lambda \begin{aligned} & \text { síndrome de Down (SD) é considerada a mais } \\ & \text { comum e antiga alteração cromossômica } \\ & \text { relacionada a uma deficiência intelectual }{ }^{1}\end{aligned}$ que gera indivíduos com características fenotípicas de fácil reconhecimento logo após o nascimento. Dentre suas principais características e condições clínicas encontram-se a hipotonia muscular, a frouxidão ligamentar, as alterações cardíacas, a protrusão de língua, os olhos arredondados e o pequeno tamanho de mãos e pés ${ }^{2,3}$.

$\mathrm{O}$ atraso no desenvolvimento de marcos motores básicos é comum nas crianças com $\mathrm{SD}$, em decorrência de seu quadro clínico, variando de indivíduo para indivíduo, sendo principalmente observado em atividades como o rolar, o alcance manual, o sentar e a marcha ${ }^{4}$. A marcha apresenta características próprias como base alargada, rotação externa de quadril e incoordenação do balanço entre os membros superiores $^{4}$. De acordo com Mancini et $\mathrm{al}^{4} \mathrm{o}$ atraso nestes marcos motores é de um ano a um ano e meio quando comparados ao desenvolvimento normal de crianças de 2 e 5 anos.

A hipotonia generalizada interfere na coordenação dos movimentos e nas reações posturais que determinam o equilíbrio e as atividades manipulativas durante uma tarefa. A frouxidão ligamentar influencia a estabilização articular e causa o aparecimento de compensações e desalinhamentos ${ }^{5}$.

Movimentos provenientes da função motora grossa são adquiridos de forma tardia, principalmente quando há aumento do nível de complexidade do movimento, já que, de acordo com Palisano et al. ${ }^{6}$ os indivíduos com SD não conseguem atingir todas as funções motoras grossas até os seis anos de idade.

Essas características motoras e intelectuais dos indivíduos com SD podem implicar em restrições em sua participação social, e seu desempenho funcional nas atividades de vida diária tornando-os dependentes de ajuda externa $^{7,8}$ em muitos momentos. Pesquisa realizada com crianças com SD de 5 a 7 anos por Volman et al. ${ }^{9}$ demonstrou maior relação do desempenho funcional avaliado pelo Inventário de Avaliação PEDI com a habilidade motora mensurada pelo Movement Assessment Battery for Children (M-ABC) e pelo Gross-Form Board (GFB) do que com o nível de capacidade mental dos participantes mensurada pelo Dutch 'Gross-Vorm Bord' Test.

Estudos apontam que as características ambientais, as barreiras e as exigências da tarefa podem, também, auxiliar no comprometimento do desempenho funcional destes ${ }^{10}$. O desempenho funcional de crianças com SD de 2 e 5 anos foi avaliado por Mancini et $\mathrm{al}^{4}$ com o uso do Inventário PEDI e constatou-se a sua baixa capacidade ao longo do desenvolvimento bem como a dependência da assistência do cuidador. A área da mobilidade que corresponde às atividades como subir e descer escadas, entrar e sair do ônibus, transferências, entre outras foi a que apresentou maior aproximação com o desempenho de crianças saudáveis, tanto na forma de realização como na independência, com o avançar da idade.

Pazin e Martins ${ }^{10}$ analisaram as habilidades funcionais de 20 crianças com SD com idades entre 2 a 8 anos por meio do Inventário PEDI e assim como Mancini et al. ${ }^{4}$ observaram escores inferiores no desempenho quando comparadas às crianças saudáveis. A área de autocuidado e função social foram as que apresentaram as limitações mais significantes. Já $\mathrm{a}$ área de mobilidade apresentou um comprometimento mais expressivo na faixa etária de 2 a 4 anos do que dos 5 aos 8 anos, ou seja, a criança apresentou melhora no desempenho com o passar dos anos. As autoras sugerem que este fato ocorra pela aquisição da habilidade de mobilidade e sua incorporação na rotina diária, reduzindo a assistência do cuidador e desenvolvendo sua independência.

Dolva et al. ${ }^{11}$ avaliaram crianças com SD aos 5 anos usando o Inventário PEDI e encontraram maior comprometimento em atividades que envolviam habilidades motoras finas, como o uso do banheiro, o manuseio com roupas, cabelos e talheres e aquelas relacionadas à área de autocuidado como o controle de esfíncter, além do baixo desempenho na área da função social. Os autores salientam que os indivíduos com SD seguem a mesma sequência do desenvolvimento motor dos indivíduos típicos, porém de uma forma mais lenta. Outra observação feita pelos pesquisadores é a qualidade da habilidade desenvolvida que não é analisada pelo Inventário de Avaliação PEDI e que se apresenta, muitas vezes, demorada e numa qualidade de aprimoramento e execução motora inferior.

Em outro estudo Dolva et al. ${ }^{7}$ compararam o desempenho funcional de 43 crianças com SD norueguesas, aos 5 e aos 7 anos de idade e notaram que estas crianças apresentavam baixo índice na aptidão das habilidades de autocuidado, como o controle urinário e intestinal e, de função social relacionada à comunicação expressiva, compreensão funcional, resolução de problemas e iniciação de afazeres domésticos, em ambas as idades. Salientaram, também, a possível correlação entre a capacidade de comunicação e cognição características da própria da síndrome com o baixo desempenho nas habilidades que envolvam resoluções de problemas e iniciativa de atividades. A interação entre os domínios autocuidado, mobilidade e função social não foi observada estatisticamente.

Leonard et al. ${ }^{12}$ avaliaram o desempenho funcional de 207 indivíduos com SD com idades entre 7 a 17 anos, de ambos os sexos, por meio da versão modificada do Functional 
Independence Measure for Children. Notaram que todas as habilidades funcionais se aprimoraram com o passar da idade e que estes necessitam de ajuda externa supervisionada para as tarefas que envolvem as habilidades de autocuidado, comunicação e atividades sociais complexas. As diferenças de gênero também foram observadas tendo o sexo feminino um melhor desenvolvimento e desempenho motor.

O conhecimento do desenvolvimento motor e a aquisição das habilidades funcionais, na linha do tempo, em indivíduos com alterações do processo neuromaturacional é de suma importância a toda equipe terapêutica envolvida com a sua evolução já que a partir desses conhecimentos possa se traçar metas terapêuticas para o seu desenvolvimento e independência funcional proporcionando melhoria da qualidade de vida e interação social, além de uma melhor compreensão das habilidades funcionais que possam ser adquiridas desses indivíduos por seus familiares.

Assim, este estudo teve como objetivo avaliar o desempenho funcional de crianças e jovens com SD em idade escolar e a interação entre o seu perfil funcional e a assistência dada pelo cuidador.

\section{PROCEDIMENTOS METODOLÓGICOS}

O estudo caracterizado como uma pesquisa transversal e descritiva foi desenvolvido em quatro Instituições de atendimento especializado às pessoas com deficiência, em cidades do interior do estado de São Paulo (Barueri, Campo Limpo Paulista, Jundiaí e Várzea Paulista). As instituições convidadas foram escolhidas por serem parceiras em pesquisas anteriores e se mostrarem interessadas em novos estudos. Todo o processo de coleta de dados ocorreu nas dependências das Instituições participantes, mediante a autorização de seus representantes legais, no período de outubro de 2010 a junho de 2011.

Foram incluídos 44 crianças e jovens com SD, atendidos pelas Instituições participantes, com idades entre 7 anos e 6 meses (90 meses) a 15 anos incompletos (179 meses), média de 10 anos (125 meses), de ambos os gêneros, sendo 23 meninos e 21 meninas. Foram critérios de exclusão a presença de alterações neurológicas e/ou ortopédicas associadas, a presença de cardiopatia grave não corrigida, ou complicações auditivas ou visuais diagnosticadas por médico que impedissem a realização das atividades descritas no Inventário.

Os principais cuidadores dos indivíduos selecionados foram convidados a participar desta pesquisa após uma explanação dos objetivos do estudo. Todos aceitaram participar da pesquisa assinando o termo de compromisso e respondendo o Inventário de Avaliação PEDI, individualmente. O projeto foi previamente aprovado pelo Comitê de Ética da
Universidade Presbiteriana Mackenzie, sob n. 1213/04/2010 e CAAE n. 0021.0.272.000-10.

O Pediatric Evaluation of Disability Inventory - PEDI, instrumento aqui utilizado para a avaliação do desempenho funcional dos indivíduos com SD é um inventário padronizado norte-americano que documenta o perfil funcional de crianças entre os 6 meses de idade aos 7 anos e 6 meses ( 90 meses) em suas atividades de vida diária. Este inventário, traduzido e adaptado para a versão brasileira ${ }^{13}$, tem como objetivo fornecer uma descrição detalhada do perfil funcional da criança, prever seu desempenho e registrar suas alterações longitudinais. De acordo com o manual ${ }^{13}$, pode ser utilizado em crianças com idades superiores aos 90 meses desde que estas apresentem desempenho funcional dentro da faixa etária avaliada pelo teste e que seja utilizado o escore contínuo para a identificação dos resultados sendo, portanto, considerável a sua utilização na população com SD que apresenta um atraso no desenvolvimento neuro-motor global ${ }^{2,4}$.

$\mathrm{O}$ instrumento foi aplicado por uma das pesquisadoras deste estudo que recebeu treinamento prévio do Inventário de Avaliação PEDI, no qual foi obtido bom índice de confiabilidade entre os participantes. As entrevistas ocorreram em lugar determinado pelas instituições, em sala reservada e individualmente, com duração aproximada de 30 minutos.

O PEDI é dividido em três partes que informam sobre: o desempenho de habilidades funcionais da criança-Parte I, a quantidade de ajuda fornecida pelo cuidador - Parte II e as modificações do ambiente físico doméstico para a rotina da criança - Parte III. Cada uma destas partes é subdividida em três áreas de função: autocuidado, mobilidade e função social.

A primeira parte do teste (Parte I) apresenta 73 itens relacionados à área de autocuidado em atividades de higiene pessoal, banho, vestir, alimentação, uso do toalete e controle de esfíncter. A área de mobilidade contem 59 itens relativos à locomoção em ambientes internos e externos, transferências e o uso de escadas. E a função social apresenta 65 atividades relacionadas à compreensão funcional, expressão funcional, resolução de problemas, brincar, auto informação, orientação temporal e proteção. Todos estes itens são avaliados numa escala de 0 a 1 , sendo o valor 0 atribuído ao item em que a criança não é capaz de executar tal atividade e o valor 1 ao item que é capaz de executar. O somatório dos escores pontuados pela criança, em cada área de função, gera o escore total.

A parte II relata a independência da criança durante suas atividades funcionais através da ajuda fornecida pelo seu cuidador. Os itens se relacionam a 8 tarefas de autocuidado, 7 de mobilidade e 5 de função social. A pontuação ocorre numa escala de 0 a 5 onde: $\mathrm{o}$ valor de zero é dado quando a criança necessita de uma assistência total do cuidador para desenvolver a atividade; o valor 1 para assistência máxima, 2 
para assistência moderada, 3 para mínima, 4 para assistência supervisionada e 5 quando a atividade é realizada de forma independente pela criança.

A terceira parte (Parte III) informa sobre a frequência das modificações do ambiente doméstico necessárias para o desempenho das atividades de vida diária do indivíduo, nas três áreas de função, em quatro categorias: nenhuma, centrada na criança, de reabilitação e extensiva.

Os dados coletados foram analisados com o auxílio do programa estatístico Minitab®. Nesta pesquisa adotouse para todos os testes de hipóteses realizados um nível de significância $\alpha$ igual a $5 \%$ sendo, portanto rejeitadas todas as hipóteses cujos testes apresentaram nível descritivo $(\mathrm{p} \leq 0,05)$. Realizou-se o teste quiquadrado de independência entre pares de variáveis para comprovar a semelhança quanto às faixas etárias nos grupos estudados. Foram realizadas análises descritivas dos dados, além de testes de aderência à distribuição Normal. Ao se comparar a média de três grupos (no caso das respostas em função da faixa etária) foram realizadas análises de variância. Quando a variável tinha distribuição Normal, foi realizada a análise de variância paramétrica que se utiliza do teste F de Fisher. No caso de rejeição da hipótese de igualdade das médias nos três grupos, foram calculados os contrastes dois a dois pelo método de Tukey. A análise de variância não-paramétrica foi realizada por meio do teste de Kruskal-Wallis. Na comparação de médias para duas amostras (grupos com síndrome de Down - GSD e grupo controle - GC) não pareadas com variâncias desconhecidas foi utilizado o teste t-de Student. Para testar a igualdade de variâncias utilizou-se o teste $\mathrm{F}$ de Fisher, quando ambas as variáveis tinham distribuição Normal, e teste de Levene, caso contrário. Foram também testadas diversas correlações lineares entre pares de variáveis aleatórias utilizando-se o coeficiente de correlação linear de Pearson.

\section{RESULTADOS}

Os resultados dos dados analisados demonstraram que na parte I do Inventário de Avaliação PEDI, referente às habilidades funcionais dos 44 participantes, somente 11 indivíduos com SD atingiram a pontuação máxima do questionário em relação à área de autocuidado, conseguindo realizar todas as atividades propostas. Nas outras duas áreas, de mobilidade e função social, os escores finais para todos os participantes se mantiveram abaixo de $100 \%$.

Na parte II, que se refere à assistência do cuidador, 9 pessoas com SD demonstraram não necessitar de ajuda do cuidador nas atividades relacionadas à área de autocuidado e 14 indivíduos com SD nas atividades que envolvem a área de mobilidade. Já na área de função social todos necessitaram do auxílio do cuidador.

Em relação à parte III, todos os cuidadores entrevistados mencionaram a falta de necessidade de modificações no ambiente domiciliar para as crianças ou jovens com SD realizarem suas atividades diárias. Sendo assim, a análise estatística da parte III do Inventário PEDI não foi necessária.

Foram verificadas as correlações entre as variáveis: habilidades funcionais de autocuidado (AC-HF), habilidades funcionais de mobilidade (Mob-HF), habilidades funcionais de função social (FS-HF), assistência do cuidador para o autocuidado (AC-Ass C), assistência do cuidador para mobilidade (Mob-Ass C) e assistência do cuidador para função social (FS-Ass C).

A correlação entre habilidades funcionais de mobilidade e assistência do cuidador para a função social mostrou-se, ao nível de 5\%, igual à zero, sendo inexistente. As demais correlações apresentaram-se positivas e significativas, evoluindo em conjunto (Figura 1).

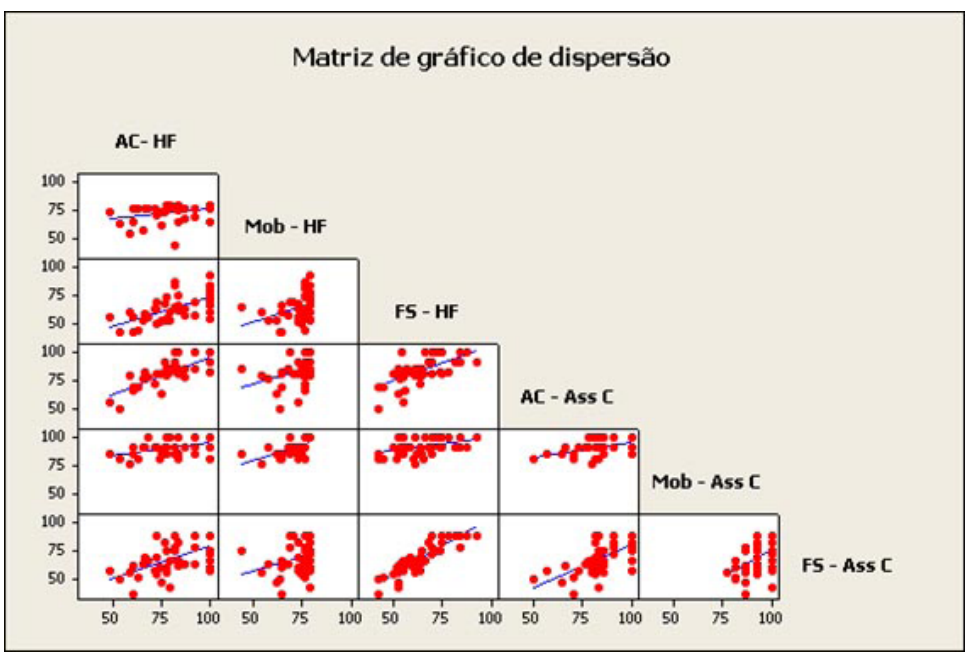

Figura 1 - Gráficos de dispersão e reta estimada de regressão simples para os Pares das variáveis em estudo 
Souza AB, et al. Caracterização do desempenho funcional de indivíduos. Rev Ter Ocup Univ São Paulo. 2015 jan./abr.;26(1):102-8.

A análise estatística entre a idade das crianças em meses e as variáveis funcionais AC-HF, Mob-HF, FS-HF, AC-Ass C, Mob-Ass C e FS-Ass C é demonstrada na Figura 2, na qual se pode observar que apenas as variáveis habilidade funcional de autocuidado, assistência do cuidador para o autocuidado e assistência do cuidador para a mobilidade apresentaram uma relação linear significante e sempre crescente com a idade.
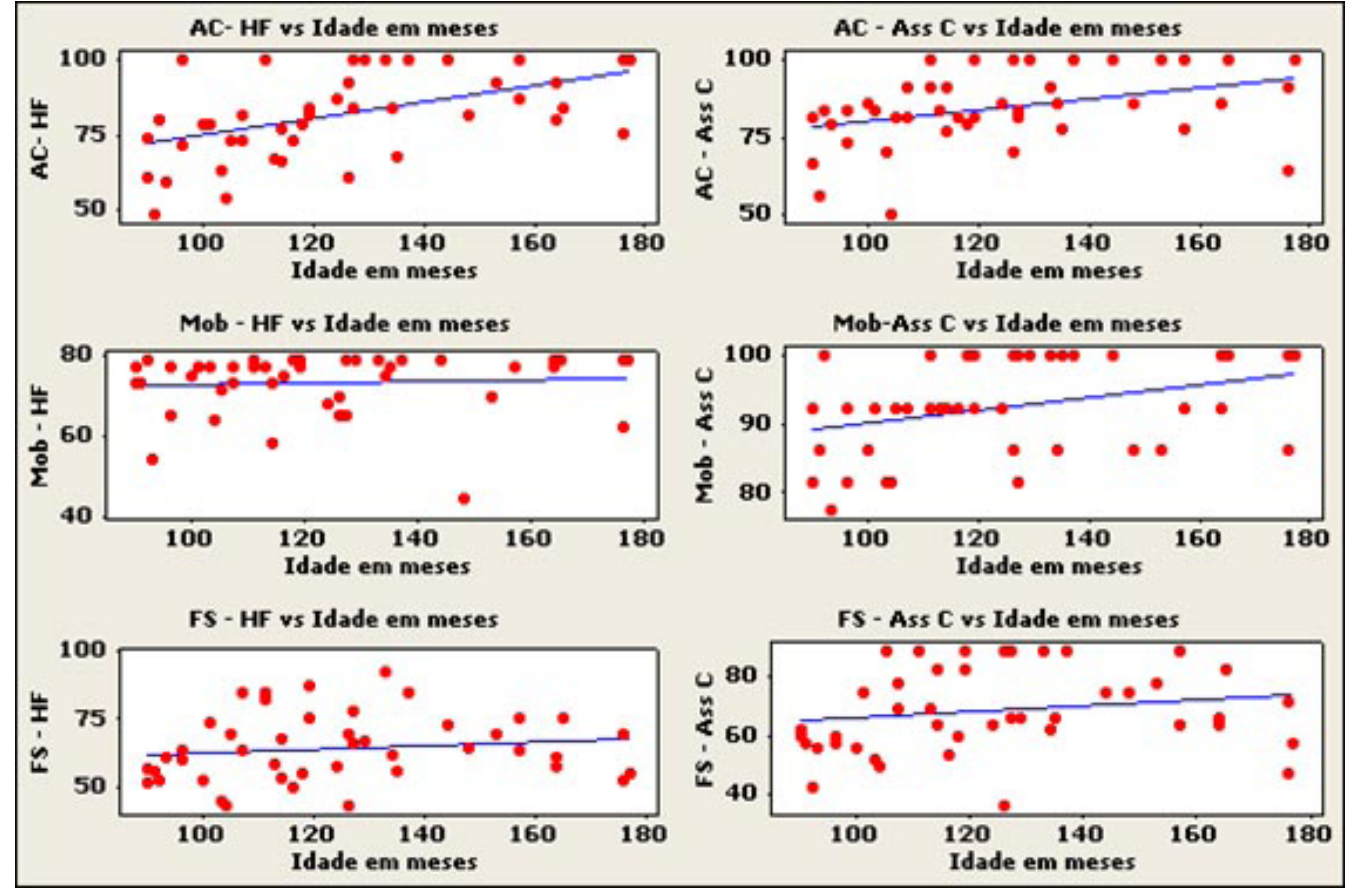

Figura 2 - Gráficos de dispersão e reta estimada de regressão simples para idade em meses das crianças e as variáveis em estudo

\section{DISCUSSÃO}

Os resultados encontrados nesse estudo indicam que o grupo estudado apresentou atrasos no desenvolvimento global mesmo com o avanço da idade, permanecendo em desvantagem em seu desempenho funcional, não atingindo os escores máximos na maioria das atividades avaliadas, da mesma forma como visto em estudos anteriores ${ }^{4,7,10,17}$.

As atividades funcionais de autocuidado como o uso do banheiro, talheres e copo, o vestir e o controle de esfíncter não foram totalmente adquiridas para o grupo estudado, já que a maioria dos participantes, ainda necessitava de ajuda externa para desempenhá-las. Este fato pode estar relacionado aos cuidados excessivos e à superproteção por parte de seus cuidadores, os quais podem tentar "facilitar" o dia a dia destas crianças ou jovens, ou apressar a realização de atividades que, mesmo que possam ser realizadas de modo independente podem levar mais tempo, já que 79,5\% do grupo estudado necessitaram da assistência do cuidador para realizar estas tarefas.

No estudo realizado por Coppede et al. ${ }^{14}$, com crianças com SD na faixa etária dos dois anos, a maioria das crianças apresentou desempenho esperado para a faixa etária para habilidades de autocuidado, embora com escores inferiores ao grupo controle composto por crianças com desenvolvimento típico. Embora o estudo tenha avaliado apenas 12 crianças, os autores relacionam este achado à suposição de que as dificuldades apresentadas em etapas posteriores, por crianças com SD em idade escolar, sejam decorrentes de limitações presentes em fases anteriores do desenvolvimento. Ressaltam ainda que as tarefas que apresentaram maiores dificuldades foram àquelas relacionadas à higiene oral, vestimenta e o uso do toalete.

Resultado semelhante ao aqui encontrado foi descrito por Silva et al. ${ }^{15}$. Os autores, que também utilizaram o inventário PEDI para avaliação, concluíram que este é um instrumento valioso para os profissionais de saúde, uma vez que identifica a área específica de desvantagem da criança avaliada, facilitando a programação de estratégias para intervenção. Os autores, que avaliaram 17 crianças com SD entre 3 e 7 anos, encontraram como resultado o desempenho funcional nas atividades de autocuidado com 
escores inferiores ao de crianças sem a síndrome. Martins et al. ${ }^{16}$ também encontraram um repertório de habilidades funcionais na área de autocuidado inferior para crianças com SD entre 6 meses e 7 anos e meio quando comprados a um grupo controle.

Para o grupo estudado, a função social foi uma das áreas que mais apresentou dificuldades, em ambas as parte analisadas (I e II), da mesma forma como encontrado em estudos anteriores ${ }^{7,10,17}$. Acredita-se que este resultado possa estar relacionado ao déficit intelectual e à dificuldade na comunicação que caracteriza o desenvolvimento da pessoa com SD, considerando-se as habilidades necessárias para o bom desempenho e pontuação nesta área no inventário PEDI.

A área da mobilidade foi a que obteve melhor pontuação e linearidade nas três áreas relacionadas às habilidades funcionais do PEDI, porém apenas 14 indivíduos $(42,2 \%)$ apresentaram independência total da assistência do cuidador. As dificuldades apresentadas podem estar relacionadas aos movimentos mais complexos desse item, como o manejo do cinto de segurança de um carro. Todavia, no estudo de Martins et $\mathrm{al}^{16}$, as áreas de mobilidade e função social, apesar de apresentarem escores menores, não registraram diferenças estatísticas entre os grupos com $\mathrm{SD}$ e controle.

A análise estatística nos mostrou que há uma relação linear entre a evolução do indivíduo com SD no desempenho de suas atividades e a assistência da cuidador, ou seja, a ajuda externa é reduzida quando a criança ou jovem com SD consegue desenvolver a atividade estudada sozinho.

Notou-se ainda progresso na capacidade funcional do grupo estudado em relação à área de autocuidado em habilidades funcionais e assistência do cuidador (partes I e II) e na área de mobilidade em assistência do cuidador (parte II).

Da mesma forma que Dolva et al. ${ }^{11}$ observou-se que a qualidade do desempenho das tarefas avaliadas não é registrada com o uso do inventário de avaliação PEDI, sendo necessários mais estudos que possibilitem o detalhamento desse aspecto e proporcionem mais informações do desenvolvimento e desempenho motor em cada faixa etária.

Com este estudo foi possível identificar a persistência de defasagens e escores mais baixos no desempenho funcional de crianças e jovens com a SD com o avanço da idade, reforçando a ideia de que os programas de estimulação e o incentivo à independência devam ser focos no planejamento educacional e terapêutico para a criança pequena e também nas etapas que se seguem na adolescência e vida adulta. Sugere-se que outras pesquisas sejam realizadas dando continuidade aos estudos com este foco para que os programas oferecidos para essas crianças e jovens sejam eficazes para a promoção do seu desenvolvimento e independência.

\section{REFERÊNCIAS}

1. Devlin L, Morrison PJ. Mosaic Down's syndrome prevalence in a complete population study. Arch Dis Child. 2004;89:11778. doi: 10.1136/adc.2003.031765.

2. Korenberg JR, Chen XN, Schipper R, Sun Z, Gonsky R, Gerwehr S, Carpenterc N, Daumer C, Dignan P, Disteche C, Graham JM, JR, Hugdins L, Mc Gillivray B, Miyazaki K, Ogasawara N, Park JP, Pagon R, Pueshel S, Sack G, Say B, Schuffenhauer S, Soukup S, Yamanaka T. Down syndrome phenotypes: The consequences of chromosomal imbalance. Proc Natl Acad Sci (USA). 1994;91:4997-5001. Available from: http://www.ncbi.nlm.nih.gov/pmc/articles/PMC43917/ pdf/pnas01133-0397.pdf.

3. Korenberg JR, Bradley C, Disteche CM. Down Syndrome: molecular mapping of the congenital heart disease and duodenal stenosis. Am J Hum Genet. 1992;50:294-302. Available from: http://www.ncbi.nlm.nih.gov/pmc/articles/
PMC1682442/pdf/ajhg00073-0054.pdf.

4. Mancini MC, Silva PC, Gonçalves SC, Martins SM. Comparação do desempenho funcional de crianças portadoras de síndrome de Down e crianças com desenvolvimento normal aos 2 e 5 anos de idade. Arq Neuropsiquiatr. 2003;61(2B):409-15. http://dx.doi.org/10.1590/S0004282X2003000300016.

5. Galli M, Rigoldi C, Brunner R, Virji-Babul N, Giorgio A. Joint stiffness and gait pattern evaluation in children with Down syndrome. Gait Posture. 2008;28:502-6. doi: 10.1016/j. gaitpost.2008.03.001.

6. Palisano, RJ, Walter SD, Russell DJ, Rosenbaum PL, Gémus M, Galuppi BE, Cunningham L. Gross motor function of children with Down syndrome: creation of motor growth curves. Arch Phys Med Rehabil. 2001;82. doi:10.1053/ apmr.2001.21956. 
Souza AB, et al. Caracterização do desempenho funcional de indivíduos. Rev Ter Ocup Univ São Paulo. 2015 jan./abr.;26(1):102-8.

7. Dolva AS, Lilja M, Hemmingsson H. Functional performance characteristics associated with postponing elementary school entry among children with Down syndrome. Am J Occup Ther. 2007;61:414-20. doi:10.5014/ajot.61.4.414.

8. Pavão SL, Silva FPS, Rocha NAC. Efeito da orientação domiciliar no desempenho funcional de crianças com necessidades especiais. Motricidade. 2011;7(1):21-9. doi: 10.6063/motricidade.7(1).117.

9. Volman MJM, Visser JJW, Lensvelt-Mulder GJLM. Functional status in 5 to 7 -year-old children with Down syndrome in relation to motor ability and performance mental ability. Disabil Rehabil. 2007;29(1):25-31. doi:10.1080/09638280600947617.

10. Pazin AC, Martins MRI. Desempenho funcional de crianças com Síndrome de Down e a qualidade de vida de seus cuidadores. Rev Neurociências. 2007;15(4):297303. Disponível em: http://revistaneurociencias.com.br/ edicoes/2007/RN\%2015\%2004/Pages \%20from\%20RN\%20 15\%2004-8.pdf.

11. Dolva AS, Coster W, Lilja M. Functional performance in children with Down syndrome. Am J Occup Ther. 2004;58:621-9. doi:10.5014/ajot.58.6.621.

Recebido para publicação: 08/10/2013

Aceito para publicação: 26/11/2014
12. Leonard S, Msall M, Brower C, Tremont M, Leonard H. Functional status of school-aged children with Down syndrome. J Paediatr Child Health. 2002;38:160-5. doi: 10.1046/j.1440-1754.2002.00736.x.

13. Mancini MC. Inventário de avaliação pediátrica (PEDI) manual da versão brasileira adaptada. Belo Horizonte: UFMG; 2005.

14. Coppede AC, Campos AC, Santos DCC, Rocha NACF. Desempenho motor fino e funcionalidade em crianças com síndrome de Down. Fisioter Pesq. 2012;19(4):363-8. http:// dx.doi.org/10.1590/S1809-29502012000400012.

15. Silva VF, Medeiros JSS, Silva, MNS, Oliveira OS, Torres RMM, Ary MLMR. Análise do desempenho de autocuidado em crianças com síndrome de Down. Cad Ter Ocup UFSCar, São Carlos. 2013;21(1):83-90. http://dx.doi.org/10.4322/ cto.2013.012.

16. Martins MRI, Fecuri MAB, Arroyo MA, Parisi MT. Avaliação das habilidades funcionais e de auto cuidado de indivíduos com síndrome de Down pertencentes a uma oficina terapêutica. Rev CEFAC, São Paulo. 2013;15(2):361-5. http:// dx.doi.org/10.1590/S1516-18462012005000088. 